\title{
Linear and/or Nonlinear Behavior of Heart Rate? A Comparative Study Using Lyapunov Exponents, Fractal Dimension, Conditional Entropy and Spectral Analysis
}

\author{
A. Ripoli, L. Zyw, C. Passino, M. Emdin \\ CNR Institute of Clinical Physiology, Pisa, Italy
}

\begin{abstract}
To investigate linear and nonlinear components of heart rate variability over the 24-hour period in healthy subjects, we analyzed RR interval series, derived from 70 recordings in free-living conditions $(37 \pm 4$ years, 34 males) by different nonlinear approaches (Largest Lyapunov Exponent -LLE- computation, quantifying exponential divergence of trajectories in the phase space; corrected conditional entropy -CCE- estimating the amount of information in the signal, and pattern fractal analysis -PFD-, able to quantify the complexity of the time series), and by autoregressive spectral analysis, too. The LLE, PFD, and CCE showed a strong correlation, whereas nonlinear parameters were significantly related even with total power, VLF, LF, and HF power of the spectra. Thus, common physiological phenomena seem to modulate both linear and nonlinear behavior of heart rate in healthy human beings.
\end{abstract}

\section{Introduction}

The analysis of heart rate variability (HRV) investigated in the frequency domain by spectral analysis is a widely used tool to determinate the neuro-hormonal control of circulation [1]. More recently, techniques derived from nonlinear dynamics and chaos theory have been adapted to quantify the dynamic behavior of the HRV [2].

In this study, linear and nonlinear analysis have been applied to circadian RR time series in order to investigate relationships between these two different approaches.

The linear approach to HRV analysis consists mainly of spectral analysis, quantifying the spectral power in different frequency ranges.

To characterize HRV nonlinear dynamics we selected different approaches: computation of the largest Lyapunov exponent (LLE), Corrected Conditional Entropy (CCE), Pattern Fractal Dimension (PFD).

\section{Nonlinear measures}

\subsection{Lyapunov exponents}

Lyapunov exponents quantify the exponential divergence of trajectories in the reconstructed phase space and sensitivity to initial conditions.

Given a $d$-dimensional phase space, there are $d$ Lyapunov exponents which are related to the evolution of the axes of an infinitesimal $d$-sphere. If $p_{\mathrm{i}}$ is the $i^{\text {th }}$ axis the $i^{\text {th }}$ exponent is defined by:

$$
\lambda_{i}=\lim _{t \rightarrow \infty} \frac{1}{t} \log _{2} \frac{p_{i}(t)}{p_{i}(0)}
$$

In our study, we considered only the largest Lyapunov exponent, defining the maximum orbit divergence.

To compute LLE from a time series, the phase space has been reconstructed from the RR interval series by the Takens method of delays $[3,4]$. We reconstructed the phase space vectors without interpolating the data choosing as sampling time the mean RR period, and using Takens formula, as if the RR series was sampled at regular intervals. The $i^{\text {th }}$ phase space vector is:

$\mathbf{y}(t)=[x(t), x(t+\tau), x(t+2 \tau), \cdots, x(t+(d-1) \tau)]$

Where $s$ is the RR series, $d$ the phase space dimension and $\tau$ the delay (multiple of the mean RR interval). LLE was computed using the Wolf algorithm [5], a set of initial conditions (two nearby vectors in the phase space) is observed over time, and LLE is evaluated by computing the divergence of the orbits (3) (see figure 1), according to: 


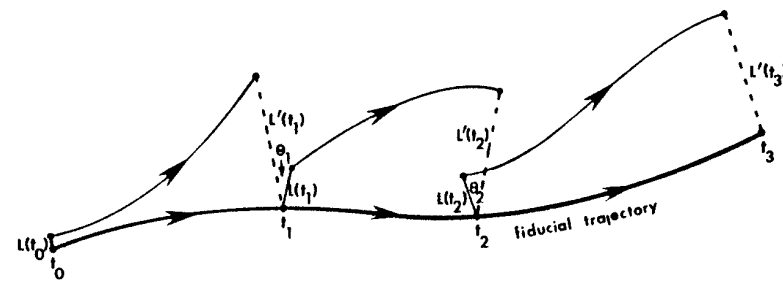

Figure 1. The algorithm follows the evolution of orbits in the phase space.

$L L E=\frac{1}{t_{M}-t_{0}} \sum_{k=1}^{M} \log _{2} \frac{L^{\prime}\left(t_{k}\right)}{L\left(t_{k-1}\right)}$

where $t_{M}-t_{0}$ is the time length of the window and $M$ the number of steps.

To verify the functionality of the algorithm it was applied to some chaotic series (logistic map, Henon map).

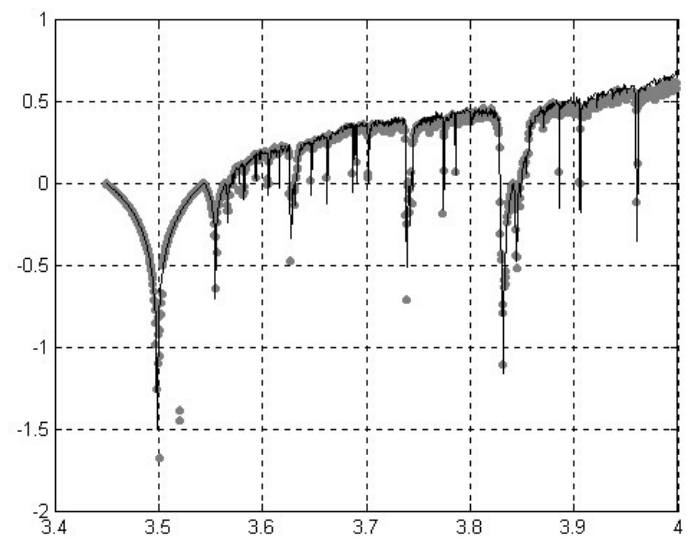

Figure 2. LLE values for the logistic map, between algorithm (gray dotted line), theoretical (black line): significant correspondence is evident.

\subsection{Fractal Dimension}

The fractal analysis of the time series was performed with an original algorithm determining Pattern Fractal Dimension (PFD) [6], PFD was derived from the classical Fractal Dimension formula (4), as defined by Katz [7],

$$
F D=\frac{\log (L)}{\log (d)}
$$

where $L$ is the length of the pattern (i.e. the sum of the distances between successive points of the broken line), $d$ is the diameter (i.e. the maximum distance between the first point and any other point of the pattern see Figure 3).

The modified pattern fractal dimension is defined as,

$$
P F D=1+\frac{\log (L / d)}{\log (n)}
$$

where $\mathrm{n}$ represents the number of samples; the measure results to be independent of linear shifts, with values always greater than 1 and smaller than 2 , as predicted by Mandelbrot [8].

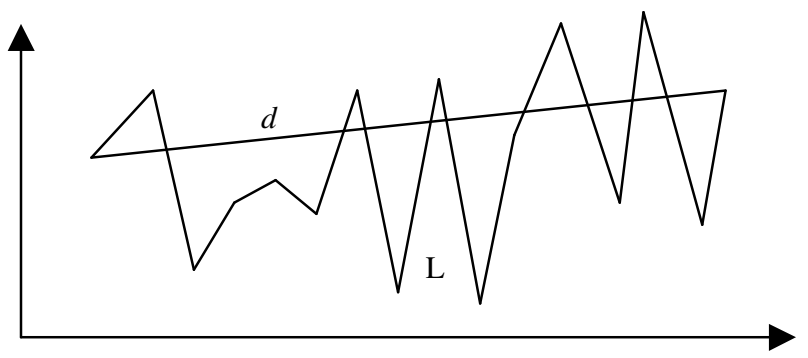

Figure 3. The diameter $d$ and the length $L$ of the pattern.

\subsection{Conditional entropy}

The recurrence of specific patterns of a given series, as an index of regularity, is quantified by the Shannon Entropy (6). Given a unitary variance and zero mean series $x(i)$, from the series a $L$-dimensional phase space is reconstructed with unitary delay, each vector y(i) represents a pattern of $L$ consecutive samples.

$$
E(L)=-\sum_{L} p_{L} \log \left(p_{L}\right)
$$

where $p_{\mathrm{L}}$ is the joint probability of the pattern $\mathrm{y}(\mathrm{i}), \mathrm{CE}$ can be represented as the variation of the SE with respect to $L$

$$
E(L / L-1)=E(L)-E(L-1)
$$

The main drawback of this technique consists of the need of long-lasting series. In order to overcome the problem related to the limited amount of data in short series and to avoid the a-priori selection of the embedding dimension the Corrected conditional Entropy (CCE) has been proposed [9], this method searches for the minimum of the function defined as: 


$$
C C E(L)=E(L / L-1)+E(1) \cdot \operatorname{perc}(L)
$$

where $E(L)$ is the estimate of the Shannon entropy of the process and $\operatorname{perc}(L)$ the percentage of length $L$ patterns found only once in the signal.
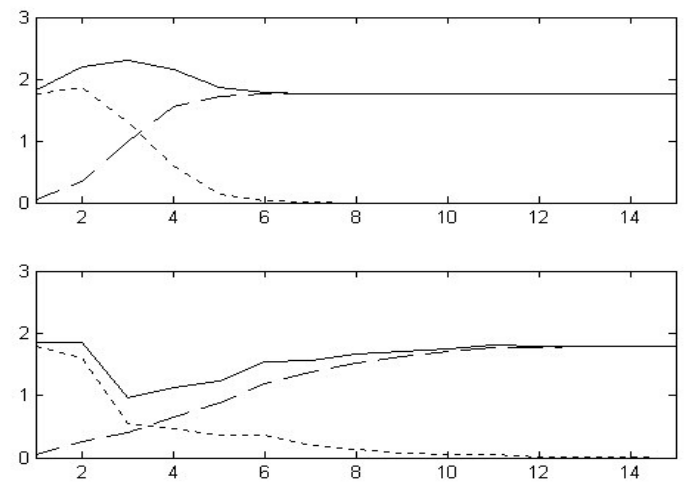

Figure 4. Values of CCE (continuous line), CE (dashed line) and perc (dotted line) for gaussian noise and $\operatorname{ar}(2)$ second order autoregressive process plotted as a function of $L$.

\section{Subjects and methods}

The ECG was digitized at $250 \mathrm{~Hz}$. The time series of RR intervals were computed throughout the 24-hour period and analyzed in consecutive intervals of 256 data points by autoregressive technique, in 70 healthy subjects (34 males, $37 \pm 4$ years, mean \pm STD).

Each extracted time series (long about 10e4 points) was analyzed over consecutive 1024-beat long windows for LLE, and 256-beat for PFD and CCE. The LLE algorithm requires the determination of some parameters: the phase space dimension $d$, the delay $\tau$. To choose the time delay $\tau$, in order to examine the nonlinear signal structure, we searched for the first minimum in the graph of average mutual information. To evaluate the embedding dimension $\mathrm{d}$, according to the method proposed by Grassberger and Procaccia [10], we determined the correlation dimension of the attractors.

According to consensus standards [1], 3 major frequency components were considered in the RR power spectrum: a very low frequency (VLF) component $(0.003$ to $0.03 \mathrm{~Hz}$ ), a low frequency (LF) component $(0.03$ to $0.15 \mathrm{~Hz}$ ), and a high frequency (HF) component $(0.15$ to $.40 \mathrm{~Hz}$ ). For each subject the different spectrum components, the total spectrum power the mean and the standard deviation on the RR-intervals time series were extracted.

The numerical results are reported as mean \pm standard deviation; correlation between measures have been investigated by means of nonparametric Spearman's $r$ correlation coefficient test. A $\mathrm{p}$ value $<.001$ was considered significant.

\section{4. $\quad$ Results}

For the RR time series considered, LLE values resulted positive $0.10 \pm 0.03$, confirming the nonlinearity of the inherent dynamical system. For the same time series, the PFD resulted 1.59 \pm 0.07 , and CCE $1.2 \pm 0.1$ (Figure 5).
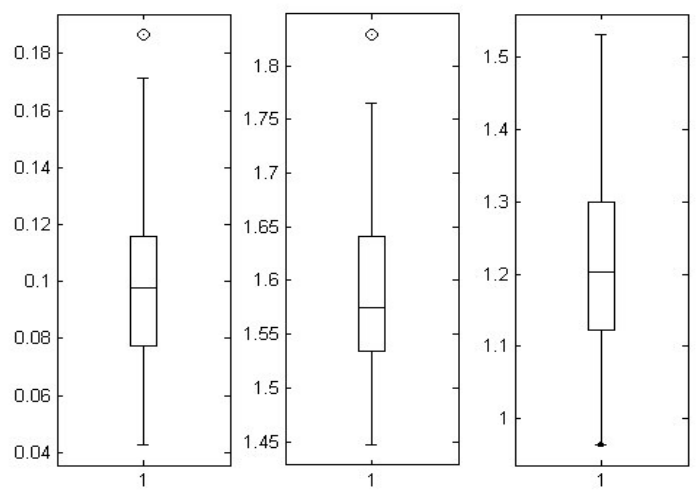

Figure 5. LLE, PFD and CCE values for the whole population.

All nonlinear indexes increase at nighttime, when a parasimpathetic shift of autonomic balance is apparent, (Figure 6).
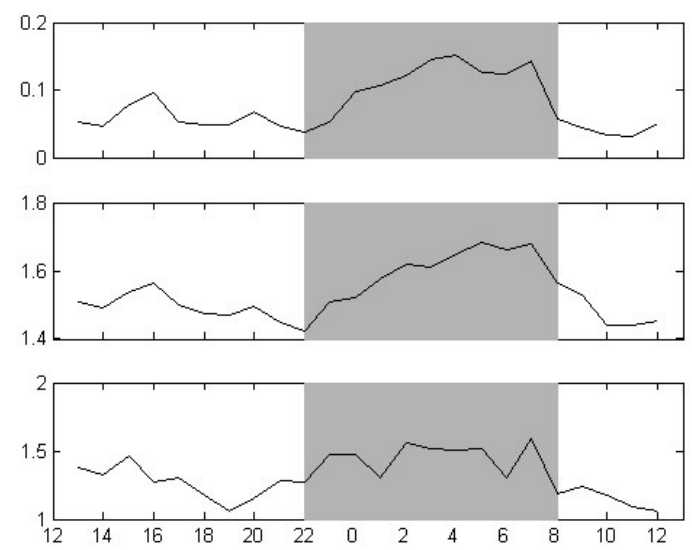

Figure 6. Boxplot represents, LLE, PFD and CCE values over the 24-hour period in one subject (gray color represents nighttime). 
Table 1. Spearman's correlation coefficients between the different measures on the population of 70 subjects. The correlation is significant $(\mathrm{p}<0.001)$ for $\mathrm{r}$ values above 0.38 .

$\begin{array}{lcccccccccc} & \text { LLE } & \text { PFD } & \text { CCE } & \text { MEAN STD } & \text { TOT } & \text { VLF } & \text { LF } & \text { HF } \\ \text { LLE } & & 0.956 & 0.802 & 0.279 & 0.565 & 0.830 & 0.321 & 0.761 & 0.839 \\ \text { PFD } & 0.956 & & 0.846 & 0.417 & 0.649 & 0.909 & 0.467 & 0.831 & 0.906 \\ \text { CCE } & 0.802 & 0.846 & & 0.520 & 0.517 & 0.738 & 0.441 & 0.679 & 0.782 \\ \text { MEAN } & 0.279 & 0.417 & 0.520 & & 0.531 & 0.483 & 0.707 & 0.477 & 0.490 \\ \text { STD } & 0.565 & 0.649 & 0.517 & 0.531 & & 0.636 & 0.556 & 0.501 & 0.705 \\ \text { TOT } & 0.830 & 0.909 & 0.738 & 0.483 & 0.636 & & 0.553 & 0.950 & 0.935 \\ \text { VLF } & 0.321 & 0.467 & 0.441 & 0.707 & 0.556 & 0.553 & & 0.549 & 0.528 \\ \text { LF } & 0.761 & 0.831 & 0.679 & 0.477 & 0.501 & 0.950 & 0.549 & & 0.808 \\ \text { HF } & 0.839 & 0.906 & 0.782 & 0.490 & 0.705 & 0.935 & 0.528 & 0.808 & \end{array}$

Regression between nonlinear and linear parameters indicated a strong linear correlation among nonlinear parameters (LLE-PFD, LLE-CCE, PFD-CCE), and between the nonlinear parameters and HF (logarithm of high frequency spectral power), LF (low frequency) and TOT (total spectral power) (Table 1).

\section{Conclusions}

This analysis points out that common mechanics, likely associated with autonomic control of sinus mode activity, determine linear and nonlinear dynamics of HRV. Furthermore, different approaches to chaotic and complex dynamics of heart rate give concordant and complementary results, confirming that the complex nature of the signal, highlighted by PFD analysis, is tightly associated with its chaotic dynamics, described by LLE approach, and with its informative content, well represented by $\mathrm{CCE}$.

\section{References}

[1] Heart rate variability: standards of measurement, physiological interpretation and clinical use. Task force of the European Society of cardiology and the North American Society of Pacing and Electrophysiology. Circulation 1996; 93: 1043-1065.

[2] Goldberger AL. Non-linear dynamics for clinicians: chaos theory, fractals, and complexity at the bedside. Lancet 1996; 347: 1312-1314.

[3] Abarbanel HDI. Analysis of observed chaotic data. Springer Verlag. Berlin, 1996.

[4] Takens F. Detecting strange attractors in turbulence. Lecture notes in mathematics No. 898 Springer, (1981).

[5] Wolf A, Swift JB, Swinney HL, Vastano JA. Determining Lyapunov exponents from a time series. Physica 16D 1985; 285-317.

[6] Ripoli A, Emdin M. Complexity of heart rate, blood pressure and respiration discolosed by pattern fractal analysis. Proceedings of Computers in Cardiology 2000,
Cambridge, Massachusetts, Vol. 27 pg. 135-138.

[7] Katz MJ. Fractals and the analysis of waveforms. Comput Biol Med 1988; 18: 145-56.

[8] Mandelbrot B. Fractal geometry of nature. Amsterdam: Freeman and C 1983.

[9] Porta A, Baselli G, Liberati D, Montano N, Cogliati C, Gnecchi-Ruscone T, Malliani A, Cerutti S Measuring regularity by means of a corrected conditional Entropy in sympathetic outflow. Biological cybernetics 1998, 78. 7178.

Address for correspondence.

Luc Zyw

Institute of Clinical Physiology

Via G. Moruzzi 1

56100 Pisa

Italy

E-mail address: luc@ifc.cnr.it 